\title{
A single-shot autocorrelator for UV femtosecond pulses
}

This content has been downloaded from IOPscience. Please scroll down to see the full text. 1990 Meas. Sci. Technol. 1637

(http://iopscience.iop.org/0957-0233/1/7/017)

View the table of contents for this issue, or go to the journal homepage for more

Download details:

IP Address: 134.76.223.157

This content was downloaded on 05/01/2017 at 13:37

Please note that terms and conditions apply.

You may also be interested in:

KrF gain module for short-pulse off-axis amplification

G Kovacs and S Szatmari

Soft x-ray brilliance of femtosecond and picosecond laser-plasmas

D Altenbernd, U Teubner, $P$ Gibbon et al.

Single-shot characterization of ultrashort light pulses

A Brun, P Georges, G Le Saux et al.

Single-shot measurement of femtosecond pulses using the optical Kerr effect

$\mathrm{H}$-S Albrecht, $\mathrm{P}$ Heist, J Kleinschmidt et al.

The generation of ultrashort laser pulses

P M W French

Longitudinally pumped quenched dye laser

J Klebniczki, S Szatmari and F P Schafer

On-line diagnostic tool for measurement of the time delay between two ultrashort light pulses $\mathrm{V}$ Tenishev, $\mathrm{V}$ Avdeichikov, A Persson et al.

Low jitter rail-gap switch triggered by sub-picosecond KrF laser pulses

G Kovacs, S Szatmari and F P Schafer

Microprocessor-based system for measurement of the characteristics of ultra-short laser pulses

F Krausz, T Juhasz, J S Bakos et al. 


\title{
A single-shot autocorrelator for uv femtosecond pulses
}

\author{
Peter Simon $\nmid \S$, Harald Gerhardt $\dagger$ and Sandor Szatmárił \\ † Laser-Laboratorium Göttingen, Im Hassel 21, D-3400 Göttingen, \\ Federal Republic of Germany \\ † Max-Planck-Institut für biophysikalische Chemie, Am Faßberg, D-3400 Göttingen, \\ Federal Republic of Germany
}

Received 30 October 1989, accepted for publication 27 February 1990

\begin{abstract}
A single-shot autocorrelator for UV pulses is presented. It uses a tilted pulse-front geometry and three-photon fluorescence of $\mathrm{XeF}$ as a non-linear detector. The readout system consists of a CCD camera and an oscilloscope. The resolution of the device is a few femtoseconds.
\end{abstract}

\section{Introduction}

Recently considerable effort has been concentrated on the development of short-pulse high-power $\mathrm{KrF}$ laser systems [1-4]. When operating these high-brightness systems, a simple shot-to-shot control of the pulse duration is important. A commonly used technique for the measurement of the pulse length at $248 \mathrm{~nm}$ is the multiple-shot autocorrelation technique, based on two-photon ionisation of NO [5-7]. However, for the recording of a multiple-shot trace, $10^{2}-10^{3}$ shots have to be accumulated, obviously limiting the feasibility of this measurement. Very recently single-shot methods have been introduced for temporal measurement of UV pulses based on two-photon fluorescence of $\mathrm{Xe}_{2}$ [8], three-photon fluorescence of $\mathrm{XeF}[9]$, and two-photon ionisation of gases $[10,11]$.

According to references [8-10] the temporal resolution of the autocorrelator is limited by the spatial resolution of the non-linear detector since a counterpropagating pulse-geometry is used. This imposes severe conditions, in addition, for the alignment of the set-up. In this respect a considerable improvement is achieved with the use of slightly tilted pulse-fronts [12] instead of counter-propagating beams, allowing easy alignment and high temporal resolution. On the other hand the detection of the spatial distribution of the ions needs a special electronic device. In reference [9] a non-linear spatial detector based on three-photon fluorescence of $\mathrm{XeF}$ is introduced where the spatial distribution of the visible fluorescence can be detected by commercial devices.

Permanent address:

\$ JATE University, Department of Experimental Physics, Dóm Tér 9 H-6720 Szeged, Hungary

| JATE University, Research Group on Laser Physics of the Hungarian Academy of Sciences, Dóm Tér 9, H-6720 Szeged, Hungary.
In this paper we present a single-shot UV autocorrelator, developed as the combination of the three-photon fluorescence method [9] and an optical arrangement using slightly tilted pulse-fronts [11]. The present device is capable of measuring pulse durations with femtosecond accuracy using a simple detection system consisting of a CCD camera and an oscilloscope.

\section{Experimental procedure}

The principle of a single-shot autocorrelator using tilted pulse-fronts (figure 1) was first reported in 1977 [12]. It can be realised by using two co-linear beams in which at least one of the pulse-fronts is tilted by a dispersive element $[13,14]$. Another way is to split the beam of the short pulse into two which are then combined at an angle

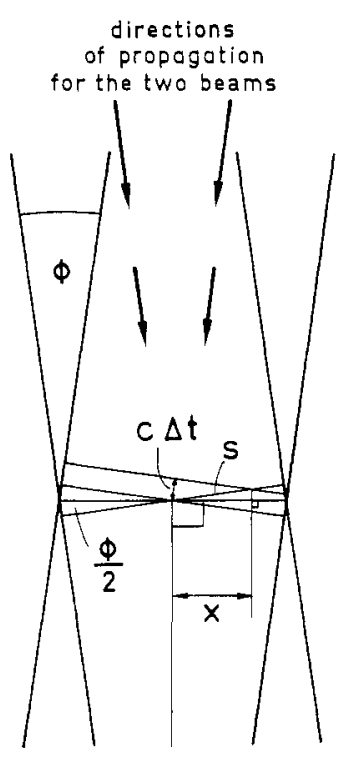

Figure 1. Scheme of the tilted pulse-front geometry for single-shot autocorrelators. 
$\varphi$ in a non-linear medium $[15,16]$. It was shown that using these configurations the spatial distribution of the response of the non-linear medium displays the autocorrelation function of the pulses along the bisector of the pulse-fronts. The required time scale can be set by proper choice of the angle $\varphi$. The advantage of the multiphoton fluorescence and ionisation technique in combination with the tilted pulse geometry is that the time window of the measurement can be changed continuously by changing the angle between the two beams [11]. In order to find the relationship between the spatial width of the observed pattern and the temporal width of the autocorrelation function, let us assume a delay of $\Delta t$ introduced into one arm. Then the maximum of the spatial pattern shifts by an amount $x$ (figure 1).

From figure 1

$$
x=s \cos \varphi / 2
$$

and

$$
\frac{c \Delta t}{s}=\sin \varphi
$$

Substituting equation (2) into (1) yields

$$
x=\frac{c}{2 \sin \varphi / 2} \Delta t
$$

When using $\mathrm{XeF}$ as a non-linear medium a cubic dependence of the $\mathrm{XeF} \mathrm{C}-\mathrm{A}$ visible fluorescence signal on the laser intensity is observed when the power density of the $248 \mathrm{~nm}$ pulse is in the range of $1 \mathrm{TW} \mathrm{cm}^{-2}$ [9]. Our laser pulses are taken from a DFDL-based $\mathrm{KrF}$ laser system [1] delivering sub-picosecond pulses of $\approx 8 \mathrm{~mJ}$ energy. An area of the beam, $6 \times 16 \mathrm{~mm}^{2}$, is selected carrying $3 \mathrm{~mJ}$ energy. Over this area the intensity is practically flat-topped, which is essential for the measurement. The estimated divergence of the beam is $\varphi \leqslant 0.4 \mathrm{mrad}$. If the beam is focused with a cylindrical lens of $f=150 \mathrm{~mm}$ along the smaller axis, one obtains a focal area of $16 \times 0.06 \mathrm{~mm}^{2}$. Since the expected pulse duration is about $300 \mathrm{fs}$ this corresponds to a power density of $\approx 1 T W\left(\mathrm{~cm}^{2}\right)$. This is just in the intensity range where generation of three-photon fluorescence in $\mathrm{XeF}$ is expected.

When a short pulse, large aperture UV beam is passed through a lens, temporal front distortion occurs $[17,18]$ resulting in a stretched pulse duration in the focus. The magnitude of this effect $(\Delta T)$ can be estimated by

$$
\Delta T=\frac{\lambda}{c} \frac{b^{2}}{8} \frac{1}{r} \frac{\mathrm{d} n}{\mathrm{~d} \lambda}
$$

where $\lambda$ is the wavelength, $c$ is the speed of light, $b$ is the smaller size of the beam at the lens, $r$ is the radius of the curved surface of the planoconvex lens and $\mathrm{d} n / \mathrm{d} \lambda$ is the dispersion of the lens material (fused silica in the experiment) [17]. In our case, equation (4) yields $27 \mathrm{fs}$. This is less than $10 \%$ of the expected pulse duration and hence an error that is of the order of the uncertainty of the pulse-width measurement. The relative contribution of the lens effects to the pulse duration does not increase even in the case of the measurement of the compressed pulses of our laser system [1]. Although the pulse duration decreases by a factor of 3-4, an increase by almost the same factor is expected in peak power. This would make possible the use of a lens of proportionally larger focal length resulting in correspondingly less pulse-front distortion (see equation (4)).

A scheme of the single-shot autocorrelator device is shown in figure 2 . The $16 \times 6 \mathrm{~mm}^{2}$ input beam is split by a $50 \%$ beam splitter. The transmitted beam is sent through a variable delay line and is directed into the $\mathrm{XeF}$ cell. The reflected beam is also directed into the XeF cell at an angle of $\varphi=2.8^{\circ}$ with respect to the other beam. Both beams are focused by the same cylindrical lens of $f=150 \mathrm{~mm}$. Focusing is done along the smaller dimension of the beams. The cell contains $100 \mathrm{kPa}$ of $\mathrm{Xe}$, $85 \mathrm{kPa}$ of $\mathrm{F}_{2}(5 \%)$ in $\mathrm{He}$, and $215 \mathrm{kPa}$ of argon as in reference [9]. The fluorescence from the focal line is observed from the side by a CCD camera (Kappa CF3). The camera-lens system serves as a UV cut filter thus selecting only the visible fluorescence from the $\mathrm{C}-\mathrm{A}$ transition of $\mathrm{XeF}$.

A typical image taken by the camera is shown in figure 3(a), obtained using a Sony UP-104 video graphic printer. The fluorescence intensity distribution is read

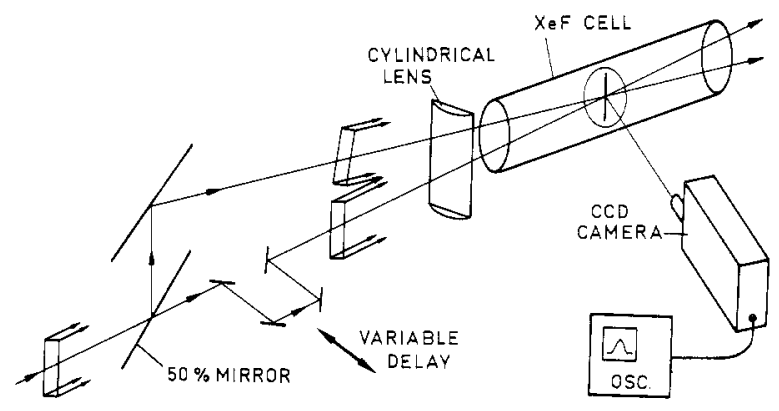

Figure 2. Experimental set-up of the autocorrelation device.

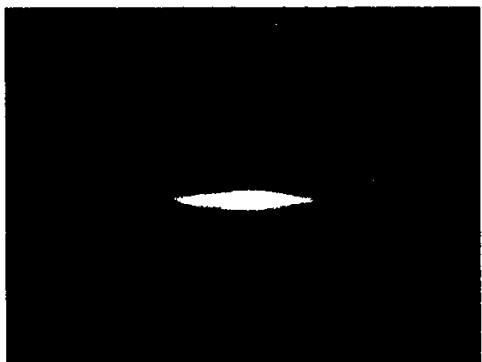

(a)

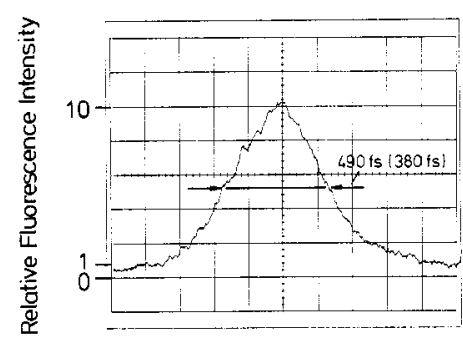

(b)

Figure 3. (a) Typical image seen by the CCD camera of the autocorrelator; $(b)$ horizontal intensity distribution of the picture: autocorrelation function. 
from a digital oscilloscope (Gould 4072) which is set to sweep along one selected line of the video frame where the intensity is the highest. A typical trace is shown in figure $3(b)$. The apparent peak-to-background ratio is close to the theoretical expectation of $10: 1$ supposing a third-order process for autocorrelation [19]. As the variable delay is changed the peak of the fluorescence shifts. This provides direct calibration of the time scale and leads to the same result as obtained in equation (3). The autocorrelation width of the pulses was typically $490 \mathrm{fs}$. The corresponding pulse duration supposing a $\operatorname{sech}^{2}(t)$ shape [9] is $380 \mathrm{fs}$.

\section{Conclusion}

In conclusion, we constructed a single-shot autocorrelator for UV pulses. The device combines tilted pulsefront geometry and the use of $\mathrm{XeF}$ as a third-order detector. Data are collected using simple commercial equipment, CCD camera and oscilloscope, easily accessible for many laboratories. The temporal resolution of the device is a few femtoseconds. Measurement of $380 \mathrm{fs}$ pulses was demonstrated.

\section{Acknowledgment}

The authors wish to thank Professor F P Schäfer for his useful comments and critical reading of the manuscript.

\section{References}

[1] Szatmári S and Schäfer F P 1988 Simplified laser system for the generation of $60 \mathrm{fs}$ pulses at $248 \mathrm{~nm}$ Opt. Commun. 69196

[2] Roberts J P, Taylor A J, Lee P H Y and Gibson R B 1988 High irradiance $248 \mathrm{~nm}$ laser system Opt. Lett. 13734

[3] Barr J M, Everall N J, Hooker C J, Ross I N, Shaw M J and Toner W T 1988 High energy amplification of picosecond pulses at $248 \mathrm{~nm}$ Opt. Commun. 66127
[4] Watanabe M, Endoh A, Sarukura $N$ and Watanabe $S$ 1989 Subpicosecond UV pulse generation for a multiterawatt $\mathrm{KrF}$ laser $A p p l$. Phys. B 48417

[5] Morita N and Yajima T 1982 A nonlinear correlation method using multiphoton ionization for the measurement of uv ultrashort pulses Appl. Phys. B 2825

[6] Rayner D M, Hackett P A and Willis C 1982 Ultraviolet laser, short pulse-width measurement by multiphoton ionization autcorrelation Rev. Sci. Instrum. $\mathbf{5 3} 537$

[7] Glownia J H, Misewich J and Sorokin P P 1986 Ultrafast ultraviolet pump-probe apparatus $J . O p t$. Soc. Am. B 31573

[8] Hutchinson M H R, McIntyre I A, Gibson G N and Rhodes C K 1987 Measurement of $248 \mathrm{~nm}$, subpicosecond pulse durations by two-photon fluorescence of xenon excimers Opt. Lett. 12102

[9] Sarukura N, Watanabe M, Endoh A and Watanabe S 1988 Single-shot measurement of subpicosecond $\mathrm{KrF}$ pulse width by three-photon fluorescence of the XeF visible transition Opt. Lett. 13996

[10 Bourne O L and Alcock A J 1986 Ultraviolet and visible single-shot autocorrelator based on multiphoton ionization Rev. Sci. Instrum. 572979

[11] Szatmári S, Schäfer F P and Jetwa J 1990 A single-shot autocorrelator for the ultraviolet with a variable time window Rev. Sci. Instrum. (submitted)

[12] Jansky J, Corradi G and Gyuzalian R N 1977 On a possibility of analysing the temporal characteristics of short light pulses Opt. Commun. 23293

[13] Wyatt R and Marinero E E 1981 Versatile single-shot background-free pulse duration measurement technique, for pulses of subnanosecond to picosecond duration Appl. Phys. 25297

[14] Szabó G, Bor Z and Müller A 1988 Phase-sensitive single-pulse autocorrelator for ultrashort laser pulses Opt. Lett. 13746

[15] Ishida Y, Yajima T and Watanabe A 1985 A simple monitoring system for single subpicosecond laser pulses using an SH spatial autocorrelation method and a CCD image sensor Opt. Commun. 5657

[16] Salin F, Georges P, Roger G and Brun A 1987 Singleshot measurement of a 52-fs pulse Appl. Opt. 264528

[17] Szatmári S and Kühnle G 1988 Pulse front and pulse duration distortion in refractive optics, and its compensation Opt. Commun. 6960

[18] Bor Z 1988 Distortion of femtosecond laser pulses in lenses and lens systems J. Mod. Opt. 351907

[19] Auston D H 1971 Higher order intensity correlations of optical pulses IEEE J. Quantum Electron. QE-7 465 